\title{
Cultura y transmisión de los derechos humanos
}

\author{
Cristina Fuertes-Planas Aleix \\ Universidad Complutense de Madrid \\ cfuertes@ccinf.ucm.es
}

Recibido: 08/10/2012

Aceptado: 23/01/2013

\begin{abstract}
Resumen
Los derechos humanos tienen que ser objeto de respeto y transmisión, al mismo tiempo que el hombre va conociendo paulatinamente las reglas morales, sociales y jurídicas. Los docentes tenemos un compromiso con la sociedad para que se conozcan y se aprenda a convivir con ellos. Es una tarea duradera, pero sin duda gratificante, cuyos resultados se irán palpando paulatinamente. Requiere conocer la naturaleza misma del hombre, su dignidad. Adentrarse en las entrañas de los distintos derechos humanos, aprender a reconocerlos como partes de nuestra cultura, fomentando su respeto y consideración.
\end{abstract}

Palabras clave: Educación, cultura, derechos humanos, dignidad humana.

\section{Culture and Transmission of Human Rights}

\begin{abstract}
Human rights must be respected and transmitted at the same time the man knows another ethic, social and legal rules. Teachers have an obligation with the society to know and to learn to life together them. That's a long but important job. Results will see slowness. It's necessary to know the man nature, his dignity. To penetrate into human rights heart, to recognize them as part of our culture, promoting his respect and consideration.
\end{abstract}

Keywords: Education, culture, human rights, human dignity.

\section{Referencia normalizada}

FUERTES-PLANAS ALEIX, Cristina (2013): “Cultura y transmisión de los derechos humanos". Estudios sobre el Mensaje Periodístico. Vol. 19, Núm. especial marzo, págs.: 187-195. Madrid, Servicio de Publicaciones de la Universidad Complutense.

Sumario: 1. Significado de educación y cultura; 1.1. Educación; 1.2. Cultura. 2. Conceptualización de los derechos humanos; 2.1. Introducción; 2.2. Aproximación a la idea de Derechos Humanos. 3. Límites de los derechos humanos. 4. Conclusiones. 5. Referencias bibliográficas.

\section{Significado de educación y cultura}

\subsection{Educación}

Educar es desarrollar o perfeccionar las facultades intelectuales, físicas y morales del niño o del joven por medio de preceptos, ejercicios y ejemplos (Diccionario RAE, voz "educar"). Supone una actitud positiva por parte del educando, quien tiene que participar de tales tareas.

La educación es el "procedimiento total mediante el cual en cualquier sociedad los adultos inculcan a los más jóvenes sus creencias, hábitos y demás valores, mientras que la enseñanza o la instrucción se refiere especialmente a la transmisión de conocimientos y a la formación intelectual". (Sentencia del Tribunal Europeo de Derechos Humanos, de 25 de febrero de 1982, caso Campbell y Cosans vs. Reino Unido).

Hemos tenido por costumbre considerar que la educación es obra casi exclusiva del entorno familiar, mientras que la instrucción, o transmisión de conocimientos y técnicas, con una participación mucho menor del educando y mucho más activa, por parte del educador, era tarea primordial de instituciones ajenas a la familia, ya fueran 
privadas o públicas. Sin embargo, en la actualidad se propugna una idea más completa, en orden a la consecución de una educación del hombre integral que responda a las necesidades cada vez más exigentes del entorno social. No cabe duda de que las modernas tecnologías, que han contribuido en gran medida a la globalización mundial, han dejado, sin embargo, a muchas personas al margen por ausencia de conocimientos en todos los niveles (Libro Blanco sobre la Educación y la Información).

La preocupación por la educación ha sido un tema recurrente a lo largo de la historia, y ha quedado patente en los clásicos de nuestra cultura, como Platón (427-347 a. C.), quien consideraba que la educación consistía en "dar al cuerpo y al alma toda la belleza y toda la perfección de que son susceptibles", o del pensamiento moderno, como Kant, que sostenía que "la educación es el desarrollo en el hombre de toda la perfección que lleve consigo su naturaleza", o en autores actuales (Moreno García y Rostán Gómez, 1974, Unidad Docente I).

Wilhem Dilthey (1833-1911) consideró que "por educación entendemos la actividad planeada mediante la cual los adultos tratan de formar la vida anímica de los seres en desarrollo" y también que "la educación no es en sí misma fin sino sólo medio para el desarrollo de una vida anímica". Para Augusto Comte (1798-1857), "la educación es la manera de aprender a vivir para otros por el hábito de hacer prevalecer la sociabilidad sobre la personalidad", "El problema general de la educación intelectual consiste en hacer que llegue, en pocos años, un solo entendimiento por lo general mediano, al mismo punto de desarrollo alcanzado, en una larga serie de siglos, por un gran número de genios superiores que aplicaron sucesivamente durante su vida entera, todas sus fuerzas al estudio de un mismo asunto”. Según Herbert Spencer (1820-1903) la educación es el proceso de preparar al hombre para la vida completa. Stuart Mill (1806-1873) expresa que "la educación es la cultura que cada generación da a la que debe sucederle, para hacerla capaz de conservar los resultados de los adelantos que han sido hechos y, si puede, llevarlos más allá". Según Durheim (1858-1917), "la educación es la acción ejercida por las generaciones adultas sobre las que todavía no están maduras para la vida social". John Dewey (1859-1952) afirma que la educación es la "suma total de procesos por medio de los cuales una comunidad o un grupo social pequeño o grande transmite su capacidad adquirida y sus propósitos con el fin de asegurar la continuidad de su propia existencia y desarrollo". Fernando de Azevedo (1894-1974) considera que la educación es un "proceso mediante el cual las generaciones adultas transmiten a las generaciones jóvenes su cultura o su tradición, para garantizar la continuidad del grupo en su calidad de todo". Y Gustavo Le Bon (1841-1931) dice que la educación "consiste en convertir lo consciente en subconsciente, esto es, formar hábitos".

Otro concepto más integral es el de William H. Kilpatrick (1871-1965): "La educación es el proceso de construcción individual que enriquece y guía la vida de tal modo que resulte más intensa en la persona y en la sociedad". O el de Raúl Bittencourt (Caracterización de los problemas filosóficos de la educación): "Proceso de adaptación progresiva de los individuos y de los grupos sociales al ambiente, por el aprendizaje valorizado, y que determina individualmente la formación de la personalidad, y socialmente la conservación y la renovación de la cultura". 
Esta preocupación se ha puesto de relieve en textos jurídicos internos e internacionales ya que, si bien el Derecho no es un vehículo directo de transmisión de educación, no cabe la menor duda de que contribuye a su expansión y desarrollo de forma definitiva.

La Constitución Española de 1978 garantiza el derecho a la educación en su artículo 27, que tiene diez apartados. Además de expresar (párrafo 4) la obligatoriedad y gratuidad de la enseñanza básica, en su párrafo 2 realiza una declaración de intenciones de gran calado:

"La educación tendrá por objeto el pleno desarrollo de la personalidad humana en el respeto a los principios democráticos de convivencia y a los derechos y libertades fundamentales."

Por otra parte, el artículo 9.2 del mismo Cuerpo legal compromete a todos los poderes públicos en este empeño, ya que la educación es el único medio para la consecución de la igualdad y libertad de todos los ciudadanos. Al mismo tiempo (en consonancia con el artículo 10.2) las normas relativas a derechos y libertades fundamentales han de ser interpretadas de acuerdo con las declaraciones internacionales ratificadas por España. Hay que hacer notar que este artículo se refiere a la dignidad del hombre, cuyo respeto es de estricta exigencia por parte de todos (gobernantes y gobernados).

"Hay que poner de relieve que estos aspectos están también consagrados a nivel internacional" (artículo 26 de la Declaración Universal de los Derechos Humanos de 10 de diciembre de 1948; Declaración de Principios de la Cooperación Cultural Internacional, de 4 de noviembre de 1966 (UNESCO), que en su artículo VII.1; Artículo 13.1 del Pacto Internacional de Derechos Económicos, Sociales y Culturales de 16 de diciembre de 1966; educación que ha de estar libre de todo tipo de discriminación, como expresa la Convención adoptada en la Conferencia General de las Naciones Unidas para la Educación, la Ciencia y la Cultura, de 14 de diciembre de 1960).

\section{Cultura}

No resulta fácil definir la noción de cultura. Cuatro son los elementos de este concepto:

1) La cultura es el producto de una formación activa de situaciones de vida

2) Esta formación es el resultado de un proceso intelectual y emocional

3) Se trata de la formación de asuntos de carácter fundamental para una sociedad

4) En un momento concreto, en el tiempo. Sin embargo, tiene una permanencia en el tiempo, continuo, perseverante, en el que la tradición juega un papel importante. Ciertos elementos objetivos compartidos (idioma, religión, Historia, etc.) conforman la existencia de una "comunidad cultural" (Arnold, 2004: 57).

La necesidad de la cultura como vehículo de manifestación de valores inherentes a la sociedad es una constante que vine repitiéndose desde Marco Tulio Cicerón (106 a. C.-43 a. C) quien acuñó el término "cultura" en su obra Disputas tusculanas. Con 
anterioridad estaba ligado a su raíz con el significado de cultivar la tierra. Este autor manifiesta que la "filosofía es cultura de la razón", enlazando la cultura con el conocimiento filosófico. Esta idea de la necesidad de la cultura ha permanecido a lo largo de la historia, haciéndose eco autores como Samuel Pufendorf (1632-1694) o Johan Goofried Von Herder (1744-1803), destacando, de forma especial las aportaciones de autores alemanes durante los siglos XIX y XX).

El proceso de educación en los derechos humanos debe propiciar la cultura de los derechos humanos. El modo a través del cual se accede a la cultura es la educación. Educar es preparar para la cultura. Por ello, es necesario que los individuos sean educados para poder entender y expresar los valores del orden vigente dentro de la comunidad en la que se vive.

Proponemos una educación e instrucción en derechos humanos que se prolongue a lo largo de la vida de la persona, conformando un ethos o cultura de los mismos. La cultura constituye el punto de encuentro de la educación y la instrucción y es, además, como hemos podido observar en las diferentes declaraciones internacionales mencionadas, una exigencia global. Los fundamentos de los derechos humanos son filosóficos, ya que son el producto de movimientos culturales milenarios. Cada profesor, cada maestro, cada institución dedicada a la enseñanza puede cumplir con la labor de difusión de los derechos humanos.

La educación es un proceso continuado de perfeccionamiento y es el resultado de dicho proceso. Se dirige a todos los aspectos de la personalidad humana, manifestaciones que emanan del orden moral, físico e intelectual. En definitiva, la educación ha de ser integral.

La educación es el modo de transmisión de la cultura. La cultura se manifiesta de diferentes modos: sistema de ideas, ciencia, arte, historia, religión, etc.

La creación de una cultura de los derechos humanos significa la implicación de una variedad de disciplinas (no sólo jurídicas) y de sectores sociales para la consecución de una cultura de la constitución (pluralismo cultural, variedad de ideas e intereses). Expresa la progresiva realización de un derecho común europeo (aunque todavía no consolidado, se va realizando a través del Derecho Comunitario Europeo, declaraciones de derechos, jurisprudencia del Tribunal de Justicia de la Comunidad Europea y del Tribunal Europeo de Derechos humanos). Para lograrlo resulta extremadamente útil el método comparatista, formado por los principios generales del Derecho de los ordenamientos estatales (Mikunda, 2009: 134 y ss.) Tiene como presupuesto y fundamento la dignidad de la persona, concepto universal, utilizado de modo igualitario, es decir, en relación con la igual dignidad del otro, bien intangible (no disponible) y absoluto que reconocemos en nosotros mismos, objeto de interpretación de la Constitución (Häberle, 2007: 171-172).

\section{Conceptualización de los derechos humanos}

\subsection{Introducción}

A ciencia cierta, no tenemos un concepto exacto sobre los derechos humanos, ya que giran en torno a valores y estos no son cuantificables. Del mismo modo que la noción de Justicia, con la que se identifican en gran medida, medimos mucho mejor la ausen- 
cia de los mismos que su presencia. Por ello, E. Bloch (1979: 141- 142), afirmó que si "desde el punto de vista de la finalidad, de la que nada sabemos, pero sí mucho de sus contenidos, si bien no sabemos qué es la salvación, qué es el bien, de una forma confiada, sin embargo sí sabemos lo suficiente como para saber lo que no es. No necesitamos dudar de que ni Nerón ni Hitler constituyen el rostro de lo humano, aunque no sepamos aún en qué consiste la riqueza o el infierno de la condición humana, pero que eso no es humano, eso sí que lo sabemos [...] a pesar de que ni conocemos el fin último en su contenido, ni se halla realizado en ninguna parte de un modo mínimo, [...] aun ignorando nosotros cuáles son de hecho los contenidos de lo utópico, sin embargo podemos saber lo que no son, y podemos saber qué es lo que frena la liberación hacia ellos."

O, en fin, con palabras de Habermas: "los derechos fundamentales, incluso en su letra y estilo, se dan a conocer como enfáticas manifestaciones de voluntad y declaraciones políticas que reaccionan contra experiencias concretas de represión y vulneración de la dignidad humana. En la mayoría de los artículos dedicados a los derechos fundamentales resuena el eco de una injusticia padecida que, por así decir, es negada palabra por palabra" (Habermas, 1998: 470).

Constatamos que las declaraciones de derechos humanos aparecen, en mayor medida, en periodos de crisis colectiva como con ocasión del surgimiento de un país (Declaración de Virginia, del 12 de junio de 1776, en lo que concierne a los Estados Unidos de Norteamérica); o con motivo de la implantación de un nuevo sistema (Carta Magna de Juan sin Tierra inglesa, de 15 de junio de 1215); o cuando se establece un orden social nuevo (Declaración de Derechos del Hombre y del Ciudadano, aprobada por la Asamblea Nacional Constituyente francesa el 26 de agosto de 1789.)

Las características generales de la protección actual de los derechos del hombre son:

a) Indivisibilidad de los derechos fundamentales y los derechos económicos y sociales

b) Derechos y libertades fundamentales absolutos o sujetos a limitaciones muy definidas y mínimas. Exigen deberes de abstención de los órganos del Estado (Estado gendarme) y ni siquiera el estado legal de excepción (en el Estado de Derecho) puede introducir limitaciones respecto a algunos de estos derechos. En la Convención Europea de Derechos Humanos, adoptada por el Consejo de Europa el 4 de noviembre de 1950 (Convenio Europeo para la protección de los derechos humanos y de las libertades Fundamentales) son inderogables absolutamente:
a) El derecho a la vida
b) Prohibición de tortura o tratamientos inhumanos o degradantes.
c) Derecho a la no retroactividad de la ley penal.

Los Pactos internacionales de las Naciones Unidas de 1966 prohíben la limitación de las libertades de pensamiento, conciencia y religión

En los derechos económicos, sociales y culturales, el sujeto aparece como titular de un crédito frente a la sociedad que exige la acción positiva del Poder, "derecho al desarrollo". La actividad del Poder exige un amplio campo de discrecionalidad que 
puede configurar nuevas formas de alienación si se prima el dato económico sobre el sociológico.

La formulación jurídica y garantía de uno y otro grupo se sujeta a dispositivos técnico-jurídicos diferentes. Los primeros se juridifican cada vez más, tanto en el plano interno como internacional, elevándolos a la categoría de derechos subjetivos alegables ante los tribunales, mientras que los otros se manifiestan como principios generales o declaraciones de intención de los Estados cuya aplicación y control corresponde a órganos políticos internos o procedimientos coactivos clásicos de derecho internacional.

\section{2. Aproximación a la idea de Derechos Humanos}

La expresión "derechos humanos" tiene las características, desde el aspecto lingüístico, de ambigüedad, vaguedad y emotividad. Y ello tanto en el lenguaje vulgar como en la praxis política, en cuanto su significación es imprecisa (Mc Keon, 1949: 37) Por esta razón se ha utilizado en numerosas ocasiones dicha expresión en determinadas luchas ideológicas, como en el lenguaje de la teoría política ética o jurídica.

Estas contradicciones se producen tanto en cuanto a su motivación (consecución de la libertad religiosa (idea mantenida por el gran jurista alemán G. Jellinek, 18511911) o, por el contrario, asegurar la propiedad privada por parte de la clase burguesa (como sostenía K. Marx, 1818-1883), como en lo que atañe a su fundamentación: iusnaturalista, es decir, los derechos humanos son anteriores a cualquier reconocimiento estatal por cuanto se fundamentan en la naturaleza humana (por ello son innatos u originarios), o positivista: los derechos humanos son los reconocidos por el Estado (Pérez Luño, 1979: 15 y ss.)

Como hemos señalado, los derechos humanos se encuentran directamente relacionados con el valor jurídico por excelencia: la Justicia. Las grandes declaraciones de derechos humanos, de tinte iusnaturalista racionalista, producto de la corriente iluminista consideraron que todos los derechos "naturales", inherentes al hombre por su propia naturaleza, conformaban un sistema normativo cerrado que respondía totalmente las exigencias de justicia. En la actualidad, el más íntimo principio de los derechos humanos es la dignidad del hombre. (Habermas, 2010: 10-25)

\section{Límites de los derechos humanos}

Los derechos humanos, sin embargo, no son absolutos sino limitados. Esos límites son, en primer lugar, intrínsecos, es decir, no pueden ser contrarios ni a su estructura, ni a su naturaleza ni a su finalidad por lo que no puede ejercitarse un derecho con perjuicio a otra persona o, simplemente, sin que exista un verdadero interés en dicho ejercicio, ya que se produciría un abuso del propio derecho.

Por lo que respecta a los límites externos de los derechos humanos serían: a) los derechos humanos de los demás, es decir, el ejercicio de nuestro derecho no puede vulnerar el contenido de otro derecho del que es titular una persona distinta. Para dilucidar esta cuestión habrá que ponderar cada uno de dichos derechos en conflicto; b) moral social, o conjunto de principios o reglas básicas que la sociedad (de forma mayoritaria) se obliga a cumplir; c) el orden público, en cuanto conjunto de condiciones de organización y funcionamiento de la sociedad para el desarrollo de la vida social 
tranquila; d) el bien común, es decir la realización de intereses generales importantes que conforman el bienestar general.

\section{Conclusiones}

En la cultura de los derechos humanos estamos implicados todos: gobernantes y gobernados; poderes políticos y ciudadanía. Es necesario difundirlos y divulgarlos, dar a conocer el catálogo de derechos para prevenir su infracción. Tienen que formar parte de la educación, desde el nacimiento, la familia, la escuela y la Universidad, lo que, sin duda, fortalecerá la democracia.

En el cincuentenario de la Declaración Universal de Derechos Humanos (19971999) el Departamento de Información Pública, Sección de desarrollo de Derechos Humanos (ONU), difundió una carta mostrando su inquietud por la educación en derechos humanos, en la que se dice:

La necesidad de fomentar la educación en la esfera de los derechos humanos se ha destacado en la Declaración Universal de Derechos Humanos y en otros documentos y tratados. En las disposiciones de esos instrumentos se señala que la educación en la esfera de los derechos humanos está encaminada a crear una cultura universal de los derechos humanos mediante la impartición de conocimientos y habilidades y la formación de actitudes. Esa educación debería estar orientada hacia:

El fortalecimiento del respeto de los derechos humanos y las libertades fundamentales; el desarrollo pleno de la personalidad humana y su sentido de dignidad; la promoción de la comprensión, la tolerancia, la igualdad entre los géneros y la amistad entre todas las naciones, las poblaciones indígenas y los grupos raciales, nacionales, étnicos, religiosos y lingüísticos; el fomento de la participación efectiva de todos en una sociedad libre; el fortalecimiento de las actividades de las Naciones Unidas para el mantenimiento de la paz. [...]

Tres aspectos se deben tener en cuenta en la promoción de los derechos humanos mediante la educación:

Los conocimientos: suministrar información acerca de los derechos humanos y los mecanismos que existen para proteger esos derechos; los valores, creencias y actitudes: promover una cultura de los derechos humanos mediante el fomento de esos procesos; la adopción de medidas: alentar a las personas a defender los derechos humanos y prevenir las violaciones de esos derechos.

Se accede a la cultura a través de los medios de comunicación, entre los que se encuentra la enseñanza, siendo el más clásico, aunque también a través de los medios de difusión de masas.

Independientemente del establecimiento de mecanismos internacionales de protección de los derechos humanos, así como de la agilización y mejora de los trámites para la formulación de quejas ante los órganos competentes, la implementación de los derechos humanos pasa por la educación en los mismos, ya que la dimensión cultural y educativa es esencial a la persona.

La educación en valores y en derechos humanos se basa, en gran medida, no sólo en el conocimiento de los mismos sino también en un programa de diálogo, debate, reflexión, cambio de opiniones en las acciones educativas, de modo que el alumno 
adquiera un bagaje intelectual, práctico, crítico de los derechos humanos, así como el respeto, empatía, sentimiento de obligatoriedad por los derechos de todos.

Naturalmente, esta labor conlleva una concienciación de los deberes y responsabilidades que todos tenemos que asumir. No existe un derecho sin un deber correlativo. Tenemos deberes cívicos y políticos, como personas particulares y como ciudadanos. Es la consecuencia de nuestro carácter social, ya que formamos parte de una comunidad en sentido amplio: familiar, regional, religiosa, cultural (nacional e internacional) y tenemos deberes y responsabilidades con ella. Sólo dentro de la comunidad se desarrolla la personalidad.

\section{Referencias bibliográficas}

ARNOLD, Rainer (2004): "La contribución de los países de la Europa Central y Oriental al desarrollo de una cultura constitucional europea", en BALAGUER CALLEJÓN, Francisco (coord.): Derecho Constitucional y Cultura. Estudios en Homenaje a Peter Häberle. Madrid, Tecnos, p. 57.

BLOCH, Ernst (1979): "El hombre del realismo utópico" [1965, traducción de J. Pérez Corral], en GÓMEZ CAFFARENA, J., y otros: A favor de Bloch. Barcelona, Libros Aisa, pp. 141-142

HÄBERLE, Peter (2007): El Estado constitucional [Estudio introductorio de Diego Valadés, traducción e índices de Héctor Fix-Hierro]. Buenos Aires, Astrea, pp. 171-172.

HABERMAS, Jürgen (1998): Facticidad y validez. Sobre el derecho y el Estado democrático de derecho en términos de teoría del discurso. Madrid, Trotta.

HABERMAS, Jürgen (2010): "El concepto de dignidad humana y la utopía realista de los derechos humanos", Diánoia, vol. LV, no. 64 (mayo 2010), pp. 3-25.

COMISIÓN DE LAS COMUNIDADES EUROPEAS (1995): Libro Blanco sobre la Educación y la Información. Enseñar y aprender, hacia la sociedad cognitiva. Bruselas

McKEON, Richard (1949): "Las bases filosóficas y las circunstancias materiales de los derechos del hombre", en UNESCO: Los Derechos del Hombre. Estudios y comentarios en torno a la nueva Declaración Universal. México-Buenos Aires, Fondo de Cultura Económica, p. 37.

MIKUNDA FRANCO, Emilio (2009): Filosofia y teoría del Derecho en Peter Häberle. Una aproximación panorámica al pensamiento del autor [Prólogo de Peter Häberle, nota de Francisco Fernández Segado]. Madrid, Dykinson Constitucional, pp. 134 y ss.

MORENO GARCÍA, José María y ROSTÁN GÓMEZ, Victoriano, (1974) [equipo docente bajo la dirección de GARCÍA HOZ, Víctor]: Introducción a las ciencias de la educación. Madrid, Universidad Nacional de Educación a Distancia (UNED), unidad didáctica 1.

PÉREZ LUÑO, Antonio Enrique (1979): Los derechos humanos. Significación, estatuto jurídico y sistema. Sevilla, Publicaciones de la Universidad de Sevilla, pp. 15 y ss. 


\section{Cristina FUERTES-PLANAS ALEIX}

Profesora contratada doctora de la Facultad de Ciencias de la Información (Sección Departamental de Filosofía del Derecho, Moral y Política I) de la Universidad Complutense de Madrid.

Secretaria Académica de la Sección Departamental de Filosofía del Derecho, Moral y política I, en la Facultad de Ciencias de la Información de la Universidad Complutense de Madrid.

Coordinadora de la Sección de Filosofía del Derecho, Moral y Política I en la Real Academia de Jurisprudencia y Legislación de Madrid.

cfuertes@ccinf.ucm.es 